\section{Perceived somatic and affective barriers for self-efficacy and physical activity}

Journal of Health Psychology 2019, Vol. 24(I3) 1850-1862 (C) The Author(s) 2017 Article reuse guidelines: sagepub.com/journals-permissions DOI: I0.1I77/I359105317705979 journals.sagepub.com/home/hpq @SAGE

\author{
Lisa M Warner', Julia K Wolff', \\ Svenja M Spuling ${ }^{2}$ and Susanne Wurm ${ }^{3}$
}

\begin{abstract}
According to Bandura's social-cognitive theory, perceptions of somatic and affective barriers are sources of self-efficacy. This longitudinal study compares general indicators of health barriers with measures of perceived somatic and affective barriers to predict self-efficacy and accelerometer-assessed physical activity in a subsample of $n=153$ (selected at random from $N=310$ ) community-dwelling German older adults. Perceived somatic and affective barriers longitudinally predicted physical activity mediated by self-efficacy, whereas general health barriers did not. Perceived health barriers to physical activity might be more important than more objective health barriers for older adults' physical activity levels.
\end{abstract}

\title{
Keywords
}

accelerometer, perceived barriers, social-cognitive theory, somatic and affective states, sources of selfefficacy, subjective health

\section{Introduction}

Being physical active on a regular basis reduces the risk of a number of lifestyle-related diseases such as coronary heart disease and type 2 diabetes, is related to a lower risk of injuries and hospitalisation due to falling, and is associated with increased psychological well-being as well as longevity - not only in younger but also in older adults (Bauman et al., 2016). Accordingly, if asked 'to what extent do you pay attention to adequate physical activity', around 45 per cent of older adults between the age of 60 and 79 years answered 'strong' or 'very strong' in a nationwide, representative health survey in Germany (Krug et al., 2013). In contrast, only around 16 per cent of older adults reach the recommended 150 minutes of moderate physical activity per week (Krug et al., 2013). The lack of compliance with physical activity recommendations - especially in older adults - is well documented in many developed countries: In Canada, 30 per cent of adults aged 65 years or above selfreport to meet recommended activity levels

\footnotetext{
'Freie Universität Berlin, Germany

${ }^{2}$ German Centre of Gerontology, Germany

${ }^{3}$ Friedrich-Alexander-Universität Erlangen-Nürnberg, Germany
}

\section{Corresponding author:}

Lisa M Warner, Division of Health Psychology, Freie Universität Berlin, Habelschwerdter Allee 45, I4I 95 Berlin, Germany.

Email: lisa.warner@fu-berlin.de 
(Ashe et al., 2008). A study from the United States, however, highlights that the proportion of older adults (60-69 years) that meet the physical activity guidelines differs depending on the methods used to assess physical activity levels - whereas 59.7 per cent self-reported to meet the guidelines, only 8.5 per cent met the guidelines when physical activity was assessed with accelerometers (Tucker et al., 2011).

Older adults report many barriers for not participating in recommended levels of physical activity, with health complaints most often reported (Dergance et al., 2003; Gellert et al., 2015; Moschny et al., 2011). Barriers such as being tired, injured or disabled, lacking vitality or perceiving pain have often been found to relate to lower levels of physical activity (Crombie et al., 2004; Rejeski et al., 2001; Salmon et al., 2003). Previous research, however, suggests that measures of individuals' perceived health status and their objective health status often do not match; moreover, indicators of subjective health become even less associated with indicators of objective health with increasing age (O'Brien Cousins, 2000; Pinquart, 2001; Wettstein et al., 2016). It is not surprising, therefore, that some studies have found physical activity levels in older adults to be better predicted by their perceived health status than their objective health problems (O’Brien Cousins and Tan, 2002; Perkins et al., 2009).

\section{The role of self-efficacy for physical activity among older adults}

Social-cognitive theory (SCT; Bandura, 1997) tries to explain this phenomenon of having sufficient physical functioning, on the one hand, and nevertheless refraining from being physically active, on the other hand, by postulating that individuals need to feel capable in order to be physically active. Individuals' subjectively perceived abilities - their self-efficacy beliefs - rather than their objective capabilities are important for initiating and maintaining activity. Bandura (1997) further proposes that especially for behaviours that require strength and stamina, such as physical activity, self-efficacy beliefs depend on individuals' interpretations and attribution of their physical capabilities. He assumes that "physiological indicators of efficacy play an especially influential role [...] in activities requiring physical strength and stamina' (Bandura, 1997: 106). According to Bandura (1997), self-efficacy and subsequent activity only decrease if an individual interprets physiological indicators such as fatigue, aches or pains as incapability for physical activity. With increasing age, the risk of facing one or multiple health problems increases (van den Bussche et al., 2011). Therefore, perceiving few somatic and affective barriers for being physically active is of particular importance to leading an active life in this age group.

Despite numerous studies on the effects of self-efficacy on physical activity in older adults (French et al., 2014; Van Stralen et al., 2009), much less empirical research has been conducted on the origins of self-efficacy. The few longitudinal studies that directly measured and compared the sources of self-efficacy for physical activity against one another came to the conclusion that mastery experiences, vicarious experiences, motivational self-talk, better subjective health and fewer negative affective states were sources of self-efficacy for physical activity (Warner et al., 2011, 2014). Another attempt to investigate the sources of self-efficacy for physical activity was undertaken in a systematic review by French and colleagues (2014). These authors classified intervention techniques to improve self-efficacy for physical activity in previous intervention studies and determined their effects on self-efficacy if these techniques were present versus absent in interventions for older adults. They found that setting graded tasks, prompting the generalisation of the behaviour, self-monitoring behavioural outcomes (e.g. improved fitness, less pain) and focusing on past successes as well as motivational interviewing were the techniques most effective to improve self-efficacy for physical activity, whereas problem solving and barrier identification were associated with lower selfefficacy after the intervention (identification of any barriers, not just health barriers, see French 
et al., 2014). Apart from the fact that subjective but not objective health as well as fewer negative states predicted self-efficacy in one longitudinal study (Warner et al., 2011) and that addressing a wide range of barriers in an intervention might decrease self-efficacy (French et al., 2014), previous research has not shed much light on the role of somatic and affective barriers, how these can be measured and whether interventionists need to consider these when they develop interventions to increase self-efficacy for physical activity among older adults.

\section{Aims of this study}

This study aimed at assessing somatic and affective barriers more explicitly than previous studies by asking participants for their perceived health barriers to physical activity and by comparing the effects of these perceived barriers to the effects of more general health measures on self-efficacy and physical activity. Based on the previous literature (e.g. Perkins et al., 2009; Warner et al., 2011), perceived (1) physical functioning, (2) pain, (3) illnesses and (4) fear of falling were chosen as subjective barriers to physical activity in old age. Building upon SCT (Bandura, 1997), it is hypothesised that (a) each of these four somatic and affective barriers will be a stronger predictor of self-efficacy than the respective more general assessment method of health issues from previous studies and (b) that the perceived somatic and affective barrier items will have stronger indirect effects on physical activity mediated via self-efficacy beliefs than their respective indicators of general health over time.

\section{Method}

\section{Participants}

A sample of community-dwelling adults aged 64 years and older was recruited via local newspapers, flyers in meeting places for older adults (e.g. senior citizens clubs), pharmacies and at general practice settings as well as via advertisement letters to members of a German health insurance company for the project PREFER II in Berlin. Ethical consent was obtained from the German Psychological Society (DGPs-SW 02_2012). The study was registered at www. clinicaltrials.gov (NCT01577134). All participants were remunerated with $25 €$. For this article, the data of all study groups were analysed as a longitudinal sample, statistically controlling for study group allocation. For more information on study groups and a CONSORT flow diagram of participants by measurement point, see Figure 2 in the Online Supplemental Material as well as Warner et al., 2016.

At Time 1, participants were on average 70 years old $(S D=4.71$, range $=64-86$ years $)$. The majority were women $(75 \%, n=106)$ and high school graduates $(65 \%, n=92)$. Participants' general fitness as assessed with the Chair Rise Test $(M=11.8)$ was equal to representative values $(M=11.8)$ of the German population at that age (Fuchs et al., 2013). Participants in this study rated their bodily pain on the Short Form-8 (SF-8) item 'How much pain have you had during the past 4 weeks?' $(M=49.38$ SF- 8 score; for further analysis, this study used the reversed score so that high scores reflect high burden of pain) slightly worse than that of German norm data $(M=53.83$; Beierlein et al., 2012). A total of eightper cent $(n=12)$ of the participants indicated to have been diagnosed with none of the conditions assessed in this study, 13 per cent $(n=20)$ had one, 33 per cent $(n=51)$ two, 14 per cent $(n=23)$ three, 16 per cent $(n=24)$ four, seven per cent $(n=10)$ five and eightper cent $(n=12)$ six or more diseases. The average number of diseases was 2.76 , which was about one condition less than a representative German sample within this age range $(M=3.6$; van den Bussche et al., 2011). Data from the representative DEGS study (German Health Interview and Examination Survey for Adults) on the number of falls within the German population aged 6579 further shows that our sample was equal in the percentage of people falling just once in 12 months; in our study 16 per cent $(n=25)$ as compared to also 16 per cent in the DEGS study. However, the PREFER II participants reported more recurrent falls, with 10 per cent $(n=15)$ of 
them indicating two or more falls as compared to six per cent in the DEGS study, which might be due to the fact that DEGS' maximum participant age was 79years (Rapp et al., 2014), whereas the current studies' age range was up to 86 years.

\section{Procedure and attrition analyses}

Eligibility criteria were assessed via self-report in an initial telephone interview. Participants were eligible if they were 64 years or older (if they turned 65 that year, which was the legal retirement age in Germany during the time of this study), self-reported to not being acutely physically impaired or disabled and not meeting the recommendation of 150 minutes moderate physical activity per week as assessed via self-report. Of a total sample of 310 participants who provided informed consent and filled in the T1 questionnaire at the German Centre of Gerontology, 153 participants were selected at random to wear an accelerometer (randomisation via the function 'sample' of the R package 'base'). $\chi^{2}$ tests and $t$-tests confirmed that the randomisation had been successful as no differences were found between participants selected to wear accelerometers and those not selected on any of the study variables and demographics measured in this study. Of these, 142 provided accelerometer data at Time 1 and 127 provided accelerometer data at Time 3,11 weeks after Time 1 (there was no accelerometer assessment conducted at Time 2, 7 weeks after Time 1). Accelerometer data attrition was due to technical and logistical problems (not enough devices), damaged or lost devices, inconveniences with wearing the device, non-compliance, forgetting and sample drop-out over time (see Figure 2 in the Supplemental Material for a CONSORT flow chart and reasons for dropout). Accelerometer data of $n=153$ could be used for the final data analyses as full information maximum likelihood estimation (FIML) was used to account for missing data (Little and Rubin, 2002).

At Time 2 and Time 3, participants received questionnaires via mail with pre-paid return envelopes. At Time 2, $136(86 \%)$ and at Time 3, 132 (84\%) participants completed the questionnaire. Participants who dropped out were compared to participants who remained in the study on all variables and demographics measured in this study by means of $\chi^{2}$ tests and $t$-tests. None of these comparisons yielded significant differences. The main self-reported reasons for dropping out were hospital admissions, serious health problems and time constraints (reasons for attrition are depicted in the Figure 2 in the Online Supplemental Material as well as Warner et al., 2016).

\section{Measures}

Dependent variable. Physical activity was assessed using GENEActiv accelerometers (Activinsights, 2016) worn around the left wrist for 10 days at Time 1 and Time 3. GENEActiv accelerometers have proven to reliably and validly assess the physical activity of older adults (Esliger et al., 2011). Due to possible reactivity biases in the first days (Motl et al., 2011) as well as technical problems and adherence problems on day 10 , data from days 1,2 and 10 were cropped in order to analyse the recommended 7-day period (Dillon et al., 2016). Therefore, activity levels from seven consecutive days (days 3-9) were transformed into moderate and vigorous minutes of physical activity per week according to validated thresholds for older adults published by Esliger et al. (2011), and summed up. There were four outliers at Time 1 and one outlier at Time 3, whose data were truncated to $2 S D$ above the mean.

Mediator. Self-efficacy for physical activity was assessed with six items (Scholz et al., 2005), two of them representing task and maintenance, and recovery self-efficacy, respectively, at Time 1 and Time 2 . The items were 'I am confident that I can exercise...' followed by '... on a regular basis even if this is difficult for me' and '.. at least two times a week for 30 minutes' (task self-efficacy), 'I am confident that I can be physically active on a long-term basis' followed by '... even though I might 
encounter difficult situations' and '... even though I might be more inclined to do something else' (maintenance self-efficacy) and 'I am confident that I can resume a physically active lifestyle' followed by '... even if I have relapsed several times' and '... even if I feel weak after a break (e.g. due to illness)' (recovery self-efficacy). Responses ranged from 1 (strongly disagree) to 4 (strongly agree) and were averaged across the six items to obtain one general self-efficacy score. Cronbach's alpha value was .83 at Time 1 and .88 at Time 2 .

\section{Independent variables}

General health barriers. The indicator for general physical functioning was the Chair Rise Test from the Physical Performance Battery at Time 1 (Guralnik et al., 1994). The Chair Rise Test is an objective measure of both lower extremity strength and balance and has been shown to indicate the onset of disability validly and reliably (Guralnik et al., 1994, 2000; Guralnik and Ferrucci, 2003). It assesses the time participants need to rise five times from a seated position (same chair, instruction and environment for each participant). Participants' arms are folded across their chests to ensure that they do not use them to get up. In this study, the time taken (in seconds) for five rises was assessed by a trained research assistant, who neither helped nor encouraged the participants. Higher scores (more seconds taken for five rises) indicate lower physical functioning.

General bodily pain was measured at Time 1 with the validated SF-8 item for bodily pain 'How much bodily pain have you had during the past 4 weeks?' (Ware et al., 2001). Ratings could be made on a 6-point scale (none, very mild, mild, moderate, severe and very severe). Answers were assigned the SF-36 v2 scale scores according to Ware and colleagues (2001) and reversed (100-score) so that higher scores reflected more severe pain in this study.

Illnesses were assessed as per a list of 15 diseases listed in the Functional Comorbidity Index, which counts diseases that cause functional limitations, at Time 1 (Groll et al., 2005). Participants could answer 'No' (0) or 'Yes' (1) to the question whether they had ever been diagnosed with any of the diseases in the provided list by a doctor. The disease list contained 14 conditions (with the percentage of presence in the accelerometer sample): arthritis (rheumatoid and osteoarthritis, $68.0 \%$ ), visual impairment (such as cataracts, glaucoma and macular degeneration, 43.1\%), hearing impairment (32.7\%), mental disorder (depression, anxiety or panic disorders, $25.5 \%$ ), osteoporosis (20.9\%), peripheral vascular disease $(19.0 \%)$, congestive heart failure (or heart disease, $13.1 \%$ ), chronic obstructive pulmonary disease (11.1\%), diabetes types I and II (9.8\%), angina $(5.2 \%)$, heart attack (myocardial infarction, $2.6 \%$ ), stroke $(4.6 \%)$, upper gastrointestinal disease $(3.9 \%)$ and neurological disease (such as multiple sclerosis or Parkinson's, 1.3\%). The 15 th condition was obesity as defined by a body mass index above 30, which was calculated by means of assessing participant's height and body weight (13.1\%).

The indicator for falls was assessed in the Time 1 questionnaire with the item 'How often have you fallen within the past 12 months? I have fallen ... times' adapted from a validated measure by Friedman and colleagues (2002).

Perceived somatic and affective barriers. Items to assess physical functioning, pain and illness as perceived somatic barriers to physical activity had been developed together with other scales to assess the sources of self-efficacy for physical activity in a previous study (Warner et al., 2014). The items were assessed at Time 1 through these statements: 'My lack of physical functioning prevents me from being physically active', 'My pain prevents me from being physically active' and 'My illnesses prevent me from being physically active'. Each item was rated on a 4-point scale from 1 (strongly disagree) to 4 (strongly agree). Items were treated as separate indicators of somatic barriers - a source of self-efficacy for physical activity - to be compared to the indicators of the general health barriers that participants reported. As these items have not yet been validated, this study also serves to assess their predictive validity for self-efficacy and physical 
activity as well as their construct validity against the more general health measures.

Fear of falling was assessed as an affective barrier to physical activity with the validated item adapted from Friedman and colleagues (2002) - 'In the past 12 months, have you been afraid that you might fall?' in the Time 1 questionnaire. Answers were given on a 5-point scale from 1 ((almost) never) to 5 ((almost) always).

Covariates. Since physical activity levels have been found to vary depending on sociodemographic factors (Ashe et al., 2008; Krug et al., 2013), statistical control variables for all analyses in the current analyses were age, sex, and education (assessed and classified according to the International Standard Classification of Education (ISCED; UNESCO, 1997), with (1) indicating low education (at most 9 years of school education), (2) indicating medium education (secondary school) and (3) indicating high education (qualifying for university admission)). Moreover, three dummy coded variables were used to control for study group allocation (see Figure 2 in the Online Supplemental Material as well as Warner et al., 2016), and whether participants had been advised to refrain from being physically active by a physician at Time 1 as well as baseline scores of self-efficacy and physical activity.

\section{Data analyses}

Descriptive statistics, correlation, attrition analyses, multicollinearity tests and tests of successful randomisation of accelerometers were performed in SPSS 23.

Path analyses with FIML were performed in Mplus 5. FIML uses all baseline information to estimate the modelled relationships to account for biases due to selective longitudinal sample attrition (Little and Rubin, 2002). Four different mediation models were estimated, each to compare the more general health barrier to its respective perceived somatic or affective barriers in predicting physical activity via self-efficacy. Indirect effects were specified and tested with
95 per cent bias-corrected confidence intervals resulting from 5000 bootstraps. Self-efficacy at Time 2 and physical activity at Time 3 were controlled for their respective baseline scores at Time 1 as well as age, sex, education, study group (three dummies) and physician advice to refrain from physical activity.

\section{Results}

Table 1 in the Online Supplemental Material shows descriptives and correlations of all variables assessed in this study. The Chair Rise Test showed a small but significant correlation with perceived physical functioning as somatic barrier to physical activity, $r=.18 \quad(p=.027)$. General bodily pain was strongly associated with perceived pain as somatic barrier to physical activity, $r=.76(p<.001)$. Illness burden showed a small but significant correlation with illnesses as perceived somatic barrier to physical activity, $r=.16(p=.046)$. The number of falls was moderately associated with the affective barrier of fear of falling, $r=.35(p<.001)$.

\section{Self-efficacy as mediator between somatic and affective barriers and physical activity}

Model I. The item to assess perceived lack of physical functioning as somatic barrier for physical activity was significantly related to self-efficacy $(b=-.19, p=.002)$ as well as indirectly to physical activity via self-efficacy $(b=-2.381$, 95\% CI $(-5.847,-.589)$ ), controlling for all above-mentioned covariates and baseline scores of self-efficacy and physical activity (see Figure 1(a)). The Chair Rise Test was associated with neither self-efficacy $(b=.01, p=.561)$ nor physical activity $(b=-.89, p=.370)$. The model fit the data well $\left(\chi^{2}=3.20, d f=2, p=.20\right.$, root mean square error of approximation (RMSEA) $=.06$, comparative fit index $(\mathrm{CFI})=.99$, standardised root mean square residual $(\mathrm{SRMR})=.01)$ and explained 32.5 per cent of the variance in selfefficacy at Time 2 and 63.3 per cent of the variance in physical activity at Time 3 , including the controls. 


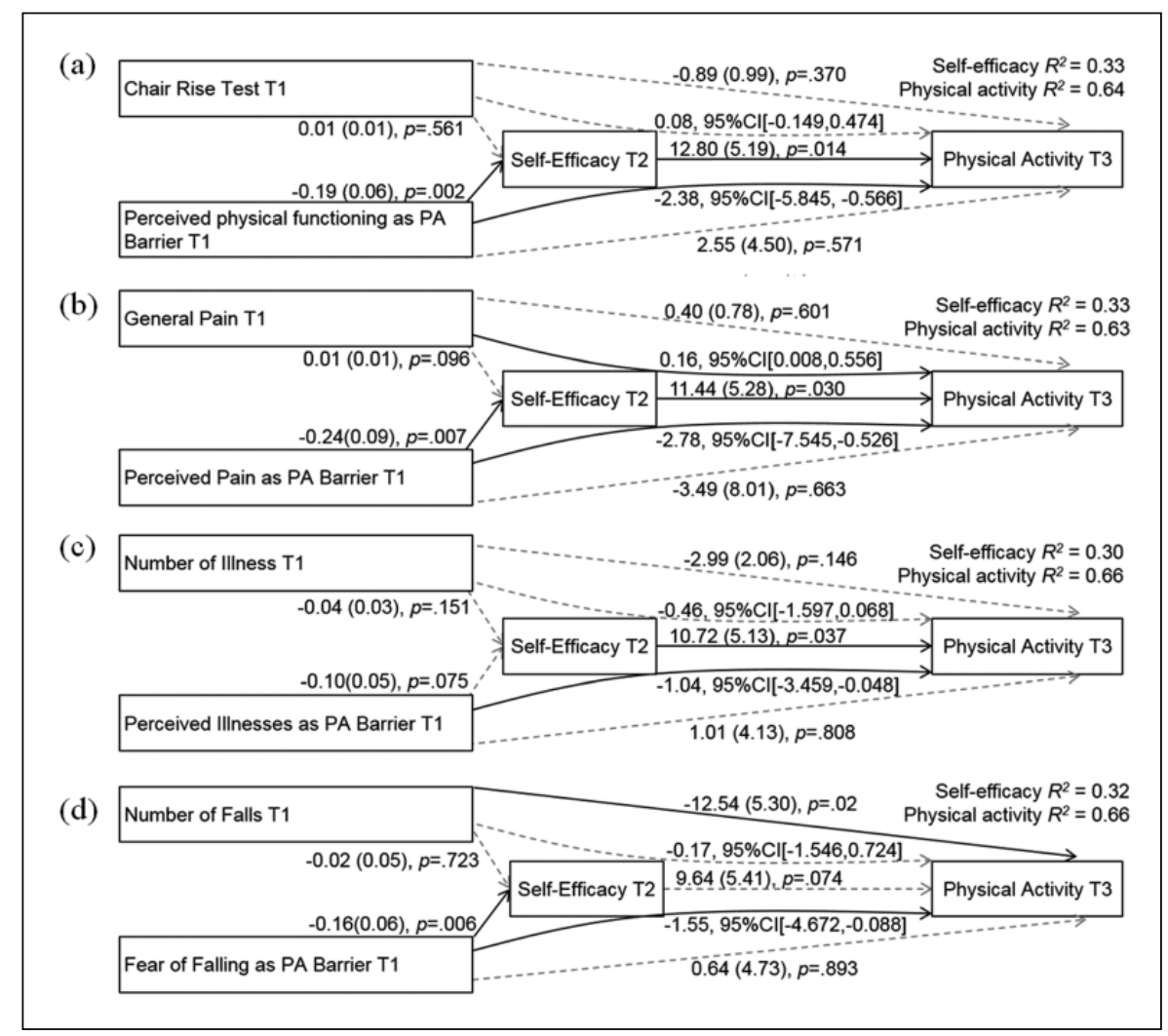

Figure I. (a-d) Path analyses predicting accelerometer-assessed moderate and vigorous physical activity minutes per week. Reported are unstandardised path coefficients with standard errors and $p$ values $(n=153)$; bias-corrected confidence intervals with 5000 bootstraps over three points in time. Statistically significant effects are illustrated in solid lines.

Note: Self-efficacy Time 2 and physical activity Time 3 were both controlled for their respective baseline scores at Time I, age, sex, education, physician advice to refrain from physical activity and three group dummies (depiction of effects for control variables omitted for clarity). Both self-efficacy Time 2 (Model a: $b=.49, S E=.09, p<.00 \mathrm{I}$; Model $b$ : $b=.5 \mathrm{I}, \mathrm{SE}=.09, p<.00 \mathrm{I}$; Model c: $b=.49, \mathrm{SE}=.10, p<.00 \mathrm{I}$; and Model $\mathrm{d}: b=.48, \mathrm{SE}=.09, p<.00 \mathrm{I})$ and physical activity Time 3 (Model a: $b=.67, \mathrm{SE}=.08, p<.00 \mathrm{I}$; Model $b: b=.68, \mathrm{SE}=.08, p<.00 \mathrm{I}$; Model $c: b=.68, \mathrm{SE}=.08, p<.00 \mathrm{I}$; and Model d: $b=.7 \mathrm{I}, S E=.08, p<.00 \mathrm{I}$ ) were significantly predicted by their statistically controlled baseline scores.

Model 2. Participants' perceptions of pain as somatic barrier to being physically active were related to self-efficacy $(b=-.24, p=.007)$ and indirectly to physical activity mediated via selfefficacy $(b=-2.78,95 \%$ CI $(-7.545,-.526))$, after controlling for covariates and baseline scores of self-efficacy and physical activity (see Figure 1(b)). General bodily pain was associated with neither self-efficacy $(b=.01, p=.096)$ nor physical activity $(b=.40, p=.601)$; however, its indirect effect via self-efficacy on physical activity was statistically significant $(b=.162,95 \%$ CI
$(.008, .556))$. This effect was very small and at the edge of being non-significant. The model fit the data well $\left(\chi^{2}=3.28, d f=2, p=.19\right.$, RMSEA $=$ $.07, \mathrm{CFI}=.99, \mathrm{SRMR}=.02)$. The total amount of variance explained in self-efficacy at Time 2 was 33.0 and 63.2 per cent in physical activity at Time 3, controlling for baseline levels of selfefficacy and physical activity and covariates. Multicollinearity statistics for perceptions of pain as a somatic barrier to being physically active and general bodily pain were acceptable in the final path model (variance inflation factors 
for both were below 10 and tolerance above .1), but the average variance inflation factors for the regression lay above 1 , indicating that the results for the pain model should be interpreted with caution (Bowerman and O'Connell, 1990).

Model 3. Neither illnesses as perceived somatic barriers to physical activity $(b=-.10, p=.08)$ nor the general illness measure $(b=-.04, p=.151)$ was significantly related to self-efficacy (see Figure 1(c)). Perceiving one's illnesses as a hindrance for physical activity nevertheless had an indirect effect on physical activity via self-efficacy $(b=-1.04,95 \% \mathrm{CI}(-3.495,-.048))$. Including all controls and baseline scores, the variance explained in self-efficacy at Time 2 was 30.04 and 65.5 per cent in physical activity at Time 3 and the model fit the data well $\left(\chi^{2}=2.46, d f=2\right.$, $p=.29, \mathrm{RMSEA}=.04, \mathrm{CFI}=1.00, \mathrm{SRMR}=.01)$.

Model 4. Participants with higher fear of falling as perceived affective barrier to physical activity had significantly lower levels of self-efficacy $(b=-.16$, $p=.006)$, which translated into lower physical activity at Time 3 via self-efficacy $(b=-1.55,95 \%$ CI (-4.672, -.088); see Figure 1(d)). The number of reported falls did not relate to self-efficacy $(b=-.02, p=.723)$, although it was associated with physical activity directly without being mediated by self-efficacy over and above controls and baseline scores of self-efficacy and physical activity $(b=-12.54, p=.018)$. After controlling for all covariates and baseline scores of self-efficacy and physical activity, fear of falling and falls, selfefficacy no longer predicted physical activity in this model $(b=9.64, p=.074)$. The model fit the data well $\left(\chi^{2}=2.98, d f=2, p=.23\right.$, RMSEA $=.06$, $\mathrm{CFI}=.99, \mathrm{SRMR}=.01)$. The amount of variance explained in self-efficacy at Time 2 was 32.0 and 65.6 per cent in physical activity at Time 3 , including baseline values of self-efficacy and physical activity and covariates.

Whereas all measures of perceived somatic barriers and the measure for affective barriers showed statistically significant indirect effects via self-efficacy on physical activity, among the more general health barriers, only the number of falls was directly associated with physical activity, and general bodily pain related to physical activity indirectly via self-efficacy (very small effect).

\section{Discussion}

According to SCT (Bandura, 1997), only if individuals interpret physiological symptoms such as pain or affective symptoms such as fear as incapability to be physically active, their self-efficacy and subsequent levels of physical activity are affected by these perceived somatic and affective barriers. This study therefore aimed at comparing general health barriers with perceived somatic and affective barriers for physical activity in older adults. As hypothesised, all perceived somatic and affective barriers had indirect effects on accelerometer-assessed physical activity, and this effect was mediated by self-efficacy beliefs (controlled for baseline values and covariates). The indicators of general health - although mostly correlated with the perceived barriers for physical activity - were unrelated to self-efficacy and did not translate into lower physical activity through a reduction in self-efficacy (with the exception of a very small indirect effect for general bodily pain).

Similar to previous research in older adults that found objective and subjective physical functioning measures to be only weakly associated or even unrelated, the correlational analyses of this study revealed that participants' objectively measured physical functioning showed only a small association with perceived physical functioning as a somatic barrier to physical activity (Pinquart, 2001; Romberg et al., 2004). In the mediation model, the comparison of a perceived lack of physical functioning (perceived somatic barrier) to physical activity and the objective measure for physical functioning (Chair Rise Test) demonstrated that participants rated their self-efficacy based on their perceived lack of physical functioning rather than on their actual physical functioning status. This perceived lack of physical functioning also translated into lower levels of subsequent physical activity. 
Based on this objective physical functioning test, our results support Bandura's (1997) assumption that individuals tend to build their self-efficacy for physical activity on their perceived and less on their actual physical functioning. This result is in line with empirical research on the sources of self-efficacy, which also showed that a measure of subjective health ('How would you describe your current health?') but not objective health (lung volume) was associated with later self-efficacy for physical activity (Warner et al., 2011). As the Chair Rise Test is a measure of lower extremity strength (Guralnik et al., 1994), participants might have taken further fitness parameters into account when they rated their perceived lack of physical functioning for physical activity, like being short of breath or unable to lift heavy weights (Crombie et al., 2004). Future research should therefore replicate this comparison by administering more comprehensive physical functioning test batteries, for example, by adding tests of gait speed and balance to the Chair Rise Test, like in the Short Physical Performance Battery (Guralnik et al., 1994).

In contrast to previous studies (Amireault et al., 2013; Wilcox et al., 2006), none of the pain measures assessed in this study were statistically significantly correlated with physical activity. Pain during physical activity is often a result of rheumatoid arthritis or osteoarthritis, which is quite common among older adults (Wilcox et al., 2006). Older adults affected by these conditions are often encouraged to be physically active to prevent further pain. This could be a possible explanation for the small correlation between pain measures and activity in our sample, in which 68.0 per cent were affected by one or both conditions and might have been active not only despite but because of their ailments (Gyurcsik et al., 2011; Wilcox et al., 2006).

In the mediation model, perceived pain as somatic barrier to activity was indirectly related to physical activity by affecting self-efficacy, whereas the more general measure of everyday pain did not affect self-efficacy or physical activity, but had an unexpected positive but very small indirect effect on physical activity via self-efficacy. This points to the notion that general pain ratings are less relevant for forming self-efficacy beliefs for physical activity than more specific measures of pain that ask for its debilitating effect specifically for physical activity. Our finding that perceptions of pain as somatic barrier to physical activity are associated with lower levels of self-efficacy, which translate into lower physical activity is in line with the assumptions of the SCT (Bandura, 1997). This finding should be cautiously interpreted due to possible multicollinearity between both pain measures.

Illnesses as assessed with an illness count based on the Functional Comorbidity Index and illnesses as assessed with the item to measure illnesses as perceived somatic barriers to physical activity were unrelated. Furthermore, the item for illnesses as perceived somatic barrier for physical activity was not significantly related to self-efficacy but was associated indirectly with subsequent physical activity via self-efficacy, whereas the count of functionally limiting illnesses did neither affect self-efficacy nor physical activity via self-efficacy. A sum score of functionally limiting illnesses might not have necessarily represented those illnesses that were most debilitating for participants' physical activity. For example, rheumatoid arthritis or osteoarthritis might have been especially hindering because of their likelihood of causing pain during exercise (Wilcox et al., 2006). Therefore, participants with diseases such as rheumatoid arthritis might have rated their illness as debilitating for physical activity, which was not reflected in the utilised illness sum score. Future studies can consider asking participants which illnesses they perceive as most debilitating for physical activity. Keeping this limitation in mind, our study found that illnesses assessed with a more general measure were not associated with self-efficacy or physical activity, whereas the perception of one's illnesses as a somatic barrier for physical activity had an indirect effect on physical activity via self-efficacy.

Fear of falling was moderately correlated with falls within the last 12 months, indicating 
that participants based their fear on former falling incidents, on the one hand. On the other hand, factors such as perceived physical and visual functioning as well as depressive symptoms and general anxiety levels might have also affected ratings of fear of falling (Friedman et al., 2002).

Whereas actual falls within the past 12 months were directly related to physical activity, but not to self-efficacy, fear of falling was associated with self-efficacy and indirectly affected later physical activity via self-efficacy. This finding corroborates the hypothesis that the affective barrier of being afraid of falling has a stronger association with self-efficacy and indirectly with physical activity than past incidences of falls. It also suggests that previous falls keep older adults from being active in the future over and above the suggested mediator of self-efficacy. These findings add to our knowledge about the association of negative affective states with self-efficacy for physical activity beyond previous research, which showed that feeling worn out, tired and tense before being physically active decreased self-efficacy levels (Warner et al., 2014). Moreover, previous research showed that fearing myocardial incidents as well as injuries increases feelings of vulnerability, which decreases the chances of becoming physically active especially in older women (O'Brien Cousins, 2000; O'Brien Cousins and Tan, 2002).

\section{Strengths and limitations}

A strength of this study was the objective assessment of physical activity with accelerometers. This was limited by the fact that - due to a limited amount of devices - not all participants could wear accelerometers. However, this was solved by a successful randomisation of devices for participants. A further advantage of this study is the longitudinal design, which allowed modelling the mediation process and the changes in self-efficacy as well as physical activity over time by controlling for selfefficacy and physical activity at baseline.

Although carefully selected, the measures applied in this study posed some limitations to the interpretation of the results. While the indicator for physical functioning status was the objective Chair Rise Test, all other indicators of general health barriers relied on self-reports. Pain is highly subjective and objective assessments make pain induction necessary, which would not represent a participant's real and everyday pain. Therefore, we relied on the bodily pain item from the SF- 8 to obtain a counterpart for pain as somatic barrier to activity. There are special pain questionnaires, which might have served the purpose to capture participants' pain better than a 1-item assessment and that might have resulted in a more distinct measure of pain to be compared to perceived pain as a barrier to physical activity (for a review on pain questionnaires for adults, see Hawker et al., 2011). For falls and illnesses, it would have been ideal to have had access to participants' medical files, which was beyond the scope of this study because of strict data protection laws in Germany. Therefore, self-reported falls and illnesses, which bear the risk of memory biases, were assessed. However, previous studies show accordance between self-reported illnesses and medical reports (Katz et al., 1996).

Since self-efficacy and the affective and somatic barriers to physical activity were assessed via self-reports in the questionnaire with 4-point Likert scales, whereas the Chair Rise Test was an objective test and the other indicators of actual health barriers had different answering formats, the congruence between the items to assess somatic and affective barriers to physical activity and self-efficacy may partly be based on shared method variance. Future research should also take fear of injuries and cardiovascular incidences into consideration when assessing affective barriers to physical activity as these were found to be particularly prevalent among older adults (Moschny et al., 2011; O'Brien Cousins, 2000). Another limitation is the current 1-item version to measure each perceived somatic and affective barrier. Future studies are advised to develop scales with multiple items for each somatic and affective barrier to reduce answering tendencies and misinterpretations of items (Lee and Bobko, 1994). 
Although we tried to recruit less motivated older adults via invitation letters that were sent to members of a large health insurance company, our sample consisted of self-selected study participants and therefore represents better-educated and healthier individuals than the average German population. Physical functioning parameters were comparable, but our sample reported lower bodily pain, more recurrent falls and fewer illnesses than what can be found in representative German samples in this age range (see the 'Participants' section). Therefore, generalisations of our results should be made with caution.

\section{Implications}

In this study, older adults' perceived somatic and affective barriers for physical activity were associated with self-efficacy and indirectly predicted physical activity over and above the effects of more general and established measures of somatic and affective barriers (physical functioning, pain, illnesses and falls indicators). This corroborates the postulation by the SCT that subjective representations of somatic and affective states are an important source of self-efficacy (Bandura, 1997).

Researchers and practitioners who would like to increase physical activity among older adults should therefore not only consider participants' objective health status but take care to additionally assess the presence of perceived somatic and affective barriers to physical activity, as these may be even more debilitating for self-efficacy beliefs than actual health problems. Activity programmes for older adults should operate within the range of an individual's actual physical ability, but should also consider integrating guidance on the interpretation and management of somatic symptoms, reassurance and supervised training to overcome fear of falling and injuries, as well as encouragement for those who perceive an initial lack in physical functioning and energy.

\section{Acknowledgements}

The authors would like to thank the German Centre of Gerontology and a team of highly motivated student assistants for helping to conduct the study.

\section{Funding}

The author(s) disclosed receipt of the following financial support for the research, authorship, and/or publication of this article: The PREFER II study (Personal Resources of Elderly People With Multimorbidity: Fortification of Effective Health Behaviour) was funded by the German Federal Ministry of Education and Research (grant number 01ET1001B).

\section{Supplemental material}

The supplemental material is available at http://journals.sagepub.com/doi/suppl/10.1177/1359105 317705979 .

\section{References}

Activinsights (2016) GENEActiv accelerometers. Available at: www.geneactiv.org

Amireault S, Godin G and Vézina-Im L-A (2013) Determinants of physical activity maintenance: A systematic review and meta-analyses. Health Psychology Review 7(1): 55-91.

Ashe MC, Miller WC, Eng JJ, et al. (2008) Older adults, chronic disease and leisure-time physical activity. Gerontology 55(1): 64-72.

Bandura A (1997) Self-Efficacy: The Exercise of Control. New York: W.H. Freeman Publishers.

Bauman A, Merom D, Bull FC, et al. (2016) Updating the evidence for physical activity: Summative reviews of the epidemiological evidence, prevalence, and interventions to promote 'active aging'. The Gerontologist 56(Suppl. 2): S268-S280.

Beierlein V, Morfeld M, Bergelt C, et al. (2012) Messung der gesundheitsbezogenen Lebensqualität mit dem SF-8 [Measuring health-related quality of life with the SF-8]. Diagnostica 58(3): 145-153 (in German).

Bowerman BL and O'Connell RT (1990) Linear Statistical Models: An Applied Approach (2nd edn). Belmont, CA: Duxbury Press.

Crombie IK, Irvine L, Williams B, et al. (2004) Why older people do not participate in leisure time physical activity: A survey of activity levels, beliefs and deterrents. Age and Ageing 33(3): 287-292.

Dergance JM, Calmbach WL, Dhanda R, et al. (2003) Barriers to and benefits of leisure time physical activity in the elderly: Differences across cultures. Journal of the American Geriatrics Society 51(6): 863-868. 
Dillon CB, Fitzgerald AP, Kearney PM, et al. (2016) Number of days required to estimate habitual activity using wrist-worn GENEActiv accelerometer: A cross-sectional study. PLoS ONE 11(5): e0109913.

Esliger DW, Rowlands AV, Hurst TL, et al. (2011) Validation of the GENEA accelerometer. Medicine and Science in Sports and Exercise 43(6): 1085-1093.

French DP, Olander E, Chisholm A, et al. (2014) Which behaviour change techniques are most effective at increasing older adults' self-efficacy and physical activity behaviour? A systematic review. Annals of Behavioral Medicine 48(2): 225-234.

Friedman SM, Munoz B, West SK, et al. (2002) Falls and fear of falling: Which comes first? A longitudinal prediction model suggests strategies for primary and secondary prevention. Journal of the American Geriatrics Society 50(8): 1329-1335.

Fuchs J, Busch M, Gößwald A, et al. (2013) Physical and cognitive capabilities among persons aged 65-79 years in Germany: Results of the German Health Interview and Examination Survey for Adults (DEGS1). Bundesgesundheitsblatt Gesundheitsforschung Gesundheitsschutz 56(5-6): 723-732.

Gellert P, Witham MD, Crombie IK, et al. (2015) The role of perceived barriers and objectively measured physical activity in adults aged 65100. Age and Ageing 44(3): 384-390.

Groll DL, To T, Bombardier C, et al. (2005) The development of a comorbidity index with physical function as the outcome. Journal of Clinical Epidemiology 58(6): 595-602.

Guralnik JM and Ferrucci L (2003) Assessing the building blocks of function: Utilizing measures of functional limitation. American Journal of Preventive Medicine 25(3 Suppl. 2): 112-121.

Guralnik JM, Ferrucci L, Pieper CF, et al. (2000) Lower extremity function and subsequent disability: Consistency across studies, predictive models, and value of gait speed alone compared with the short physical performance battery. The Journals of Gerontology: Series A 55(4): 221-231.

Guralnik JM, Simonsick EM, Ferrucci L, et al. (1994) A short physical performance battery assessing lower extremity function: Association with self-reported disability and prediction of mortality and nursing home admission. Journals of Gerontology 49(2): M85-M89.

Gyurcsik NC, Brawley LR, Spink KS, et al. (2011) Is level of pain acceptance differentially related to social cognitions and behavior? The case of active women with arthritis. Journal of Health Psychology 16(3): 530-539.

Hawker GA, Mian S, Kendzerska T, et al. (2011) Measures of adult pain: Visual Analog Scale for Pain (VAS Pain), Numeric Rating Scale for Pain (NRS Pain), McGill Pain Questionnaire (MPQ), Short-Form McGill Pain Questionnaire (SF-MPQ), Chronic Pain Grade Scale (CPGS), Short Form-36 Bodily Pain Scale (SF-36 BPS), and Measure of Intermittent and Constant Osteoarthritis Pain (ICOAP). Arthritis Care \& Research 63(Suppl. 11): 240-252.

Katz JN, Chang LC, Sangha O, et al. (1996) Can comorbidity be measured by questionnaire rather than medical record review? Medical Care 34(1): 73-84.

Krug S, Jordan S, Mensink GBM, et al. (2013) Physical activity: Results of the German Health Interview and Examination Survey for Adults (DEGS1). Bundesgesundheitsblatt Gesundheitsforschung Gesundheitsschutz 56(5-6): 765-771.

Lee C and Bobko P (1994) Self-efficacy beliefs: Comparison of five measures. Journal of Applied Psychology 79(3): 364-369.

Little RJA and Rubin DB (2002) Statistical Analysis with Missing Data. New York: Wiley.

Moschny A, Platen P, Klaassen-Mielke R, et al. (2011) Barriers to physical activity in older adults in Germany: A cross-sectional study. International Journal of Behavioral Nutrition and Physical Activity 8(1): 121.

Motl RW, McAuley E and Dlugonski D (2011) Reactivity in baseline accelerometer data from a physical activity behavioral intervention. Health Psychology 32(2): 172-175.

O'Brien Cousins S (2000) 'My heart couldn't take it': Older women's beliefs about exercise benefits and risks. The Journals of Gerontology: Series B 55(5): 283-294.

O'Brien Cousins S and Tan M (2002) Sources of efficacy for walking and climbing stairs among older adults. Physical \& Occupational Therapy in Geriatrics 20(3): 51-68.

Perkins HY, Baum GP, Taylor CLC, et al. (2009) Effects of treatment factors, comorbidities and health-related quality of life on self-efficacy for 
physical activity in cancer survivors. PsychoOncology 18(4): 405-411.

Pinquart M (2001) Correlates of subjective health in older adults: A meta-analysis. Psychology and Aging 16(3): 414-426.

Rapp K, Freiberger E, Todd C, et al. (2014) Fall incidence in Germany: Results of two populationbased studies, and comparison of retrospective and prospective falls data collection methods. BMC Geriatrics 14: 105-105.

Rejeski WJ, Shelton B, Miller M, et al. (2001) Mediators of increased physical activity and change in subjective well-being: Results from the activity counseling trial (ACT). Journal of Health Psychology 6(2): 159-168.

Romberg A, Virtanen A, Aunola S, et al. (2004) Exercise capacity, disability and leisure physical activity of subjects with multiple sclerosis. Multiple Sclerosis 10(2): 212-218.

Salmon J, Owen N, Crawford D, et al. (2003) Physical activity and sedentary behavior: A populationbased study of barriers, enjoyment, and preference. Health Psychology 22(2): 178-188.

Scholz U, Sniehotta FF and Schwarzer R (2005) Predicting physical exercise in cardiac rehabilitation: The role of phase-specific self-efficacy beliefs. Journal of Sport \& Exercise Psychology 27(2): 135-151.

Tucker JM, Welk GJ and Beyler NK (2011) Physical activity in U.S. adults: Compliance with the physical activity guidelines for Americans. American Journal of Preventive Medicine 40(4): 454-461.

UNESCO (1997) International standard classification of education. Available at: www.uis.unesco.org/publications/ISCED97

Van den Bussche H, Schön G, Kolonko T, et al. (2011) Patterns of ambulatory medical care utilization in elderly patients with special reference to chronic diseases and multimorbidity Results from a claims data based observational study in Germany. BMC Geriatrics 11: 54.

Van Stralen MM, De Vries H, Mudde AN, et al. (2009) Determinants of initiation and maintenance of physical activity among older adults: A literature review. Health Psychology Review 3(2): 147-207.

Ware J, Kosinski M, Dewey J, et al. (2001) How to Score and Interpret Single-Item Health Status Measures: A Manual for Users of the SF-8 Health Survey. Lincoln, RI: Quality-Metric Incorporated.

Warner LM, Schüz B, Knittle K, et al. (2011) Sources of perceived self-efficacy as predictors of physical activity in older adults. Applied Psychology: Health and Well-Being 3(2): 172-192.

Warner LM, Schüz B, Wolff JK, et al. (2014) Sources of self-efficacy for physical activity. Health Psychology 33(11): 1298-1308.

Warner LM, Wolff JK, Ziegelmann JP, et al. (2016). Revisiting self-regulatory techniques to promote physical activity in older adults: Nullfindings from a randomised controlled trial. Psychology \& Health, 31(10), 1145-1165.

Wettstein M, Schilling OK and Wahl H-W (2016) 'Still feeling healthy after all these years': The paradox of subjective stability versus objective decline in very old adults' health and functioning across five years. Psychology and Aging 31(8): 815-830.

Wilcox S, Der Ananian C, Abbott J, et al. (2006) Perceived exercise barriers, enablers, and benefits among exercising and nonexercising adults with arthritis: Results from a qualitative study. Arthritis Care \& Research 55(4): 616-627. 